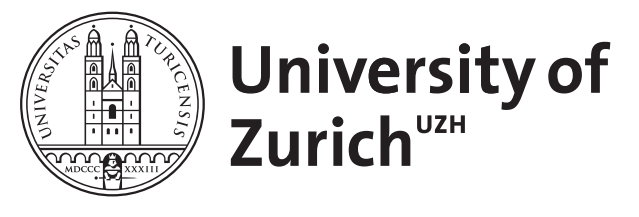

\title{
How people use the web in large indoor spaces
}

\author{
Ren, Yongli ; Tomko, Martin ; Ong, Kevin ; Sanderson, Mark
}

\begin{abstract}
We report a preliminary study of mobile Web behaviour in a large indoor retail space. By analysing a Web log collected over a 1 year period at an inner city shopping mall in Sydney, Australia, we found that 1) around $60 \%$ of registered Wi-Fi users actively browse the Internet, and the rest $40 \%$ do not, with around $10 \%$ of these users using Web search engines. Around $70 \%$ of this Web activity in the investigated mall come from frequent visitors; 2) the content that indoor users search for is different from the content they consume while browsing; 3) the popularity of future indoor search queries can be predicted with a simple theoretical model based on past queries treated as a weighted directed graph. The work described in this paper underpins applications such as the prediction of users' information needs, retail recommendation systems, and improving the mobile Web search experience.
\end{abstract}

DOI: https://doi.org/10.1145/2661829.2661929

Posted at the Zurich Open Repository and Archive, University of Zurich

ZORA URL: https://doi.org/10.5167/uzh-101132

Conference or Workshop Item

Published Version

Originally published at:

Ren, Yongli; Tomko, Martin; Ong, Kevin; Sanderson, Mark (2014). How people use the web in large indoor spaces. In: CIKM '14: Proceedings of the 23rd ACM International Conference on Conference on Information and Knowledge Management, Shanghai (China), 3 November 2014 - 7 November 2014. The ACM Guide to Computing Literature, 1879-1882.

DOI: https://doi.org/10.1145/2661829.2661929 


\title{
How People Use the Web in Large Indoor Spaces
}

\author{
Yongli Ren ${ }^{1}$, Martin Tomko ${ }^{2}$, Kevin Ong ${ }^{1}$, Mark Sanderson ${ }^{1}$ \\ ${ }^{1}$ School of Computer Science and Information Technology, RMIT University, Melbourne, Australia \\ ${ }^{2}$ Department of Computing and Information Systems, the University of Melbourne, Melbourne, Australia \\ yongli.ren@rmit.edu.au, tomkom@unimelb.edu.au, kevin.ong@rmit.edu.au, \\ mark.sanderson@rmit.edu.au
}

\begin{abstract}
We report a preliminary study of mobile Web behaviour in a large indoor retail space. By analysing a Web log collected over a 1 year period at an inner city shopping mall in Sydney, Australia, we found that 1) around $60 \%$ of registered Wi-Fi users actively browse the Internet, and the rest $40 \%$ do not, with around $10 \%$ of these users using Web search engines. Around $70 \%$ of this Web activity in the investigated mall come from frequent visitors; 2) the content that indoor users search for is different from the content they consume while browsing; 3) the popularity of future indoor search queries can be predicted with a simple theoretical model based on past queries treated as a weighted directed graph. The work described in this paper underpins applications such as the prediction of users' information needs, retail recommendation systems, and improving the mobile Web search experience.
\end{abstract}

\section{Categories and Subject Descriptors}

H.3.3 [Information Search and Retrieval]: Search process; H.1.2 [User/Machine Systems]: [Human factors]

\section{General Terms}

Experimentation

\section{Keywords}

Indoor mobile Web behaviour, query popularity propagation

\section{INTRODUCTION}

Large-scale indoor spaces are increasingly equipped with free Internet via $\mathrm{Wi}-\mathrm{Fi}$, the use of which can be logged and studied. With such infrastructure in place, it is possible to collect a variety of parameters about user behaviour. These environments are visited on a regular basis by a large number of visitors. For instance, The Mall in Dubai attracted 75 million visitors in 2013 [2]. Tracking the location of users in

Permission to make digital or hard copies of all or part of this work for personal or classroom use is granted without fee provided that copies are not made or distributed for profit or commercial advantage and that copies bear this notice and the full citation on the first page. Copyrights for components of this work owned by others than ACM must be honored. Abstracting with credit is permitted. To copy otherwise, or republish, to post on servers or to redistribute to lists, requires prior specific permission and/or a fee. Request permissions from Permissions@ acm.org.

CIKM'14, November 03 - 07 2014, Shanghai, China

Copyright 2014 ACM 978-1-4503-2598-1/14/11 ...\$15.00.

http://dx.doi.org/10.1145/2661829.2661929. time and space along with their Web activity allows for the study of their needs, establishing whether these are appropriately supported by the environments, and exploring how the indoor spaces cope with the presence of diverse visitors; an important consideration in, for example, hospitals [11].

An indoor space imposes a range of social, technical, and physical constraints. While Web query logs have been widely studied, both on desktop machines $[9,5]$ and on mobile devices $[12,7,4,8]$, there are few published studies that analyse Web search in large-scale indoor spaces. By analysing a log of Web activities of more than 120,000 users in over 1 year period, we study how people behave on the Web in indoor spaces and whether information access trends can be predicted. Such predictions have practical applications, e.g. for predicting consumer behaviour.

In this paper, we address the following questions:

- How do people behave on the Web in large indoor retail spaces? (Section 3)

- What is the indoor Web search behaviour? (Section 4)

- Can the popularity of indoor Web search queries be predicted? (Section 5)

\section{DATA ACQUISITION}

We study an anonymized dataset of internet accesses taken from a free Wi-Fi network operated by a large inner-city shopping mall. The mall is covered by around $70 \mathrm{Wi}-\mathrm{Fi} \mathrm{Ac}-$ cess Points (AP). The dataset includes three kinds of logs: a Wi-Fi Access-point association Log (AL), a Web Browsing Log (BL) and a Web Query Log (QL), collected between September 2012 and October 2013. Table 1 shows summary statistics of the three logs. For this research, all user identifiable information was replaced by a hash key in an non-invertible way.

Only devices connected to the free Wi-Fi provided by the mall are logged. The logs do not track users but rather mobile devices through the device's Wi-Fi MAC address, which was replaced by a hash key. Thus, there is no ground truth about the identities of the users (e.g. shoppers or mall employees). The term Users is used to refer to unique devices appearing in AL, a subset of such users are browsers who appear in the BL, and searchers are those users who appear in the QL. The BL includes traffic to the Web originating from the mobile Web browser, as well as from other apps. Traffic from apps currently cannot be easily filtered out. The QL was extracted from the BL (it is a subset of $\mathrm{BL}$ ), by isolating searches pointing to search engines, including Google(110148, 92.4\% of QL), Yahoo (6915, 5.8\% 
Table 1: Summary statistics of the AL, BL and QL.

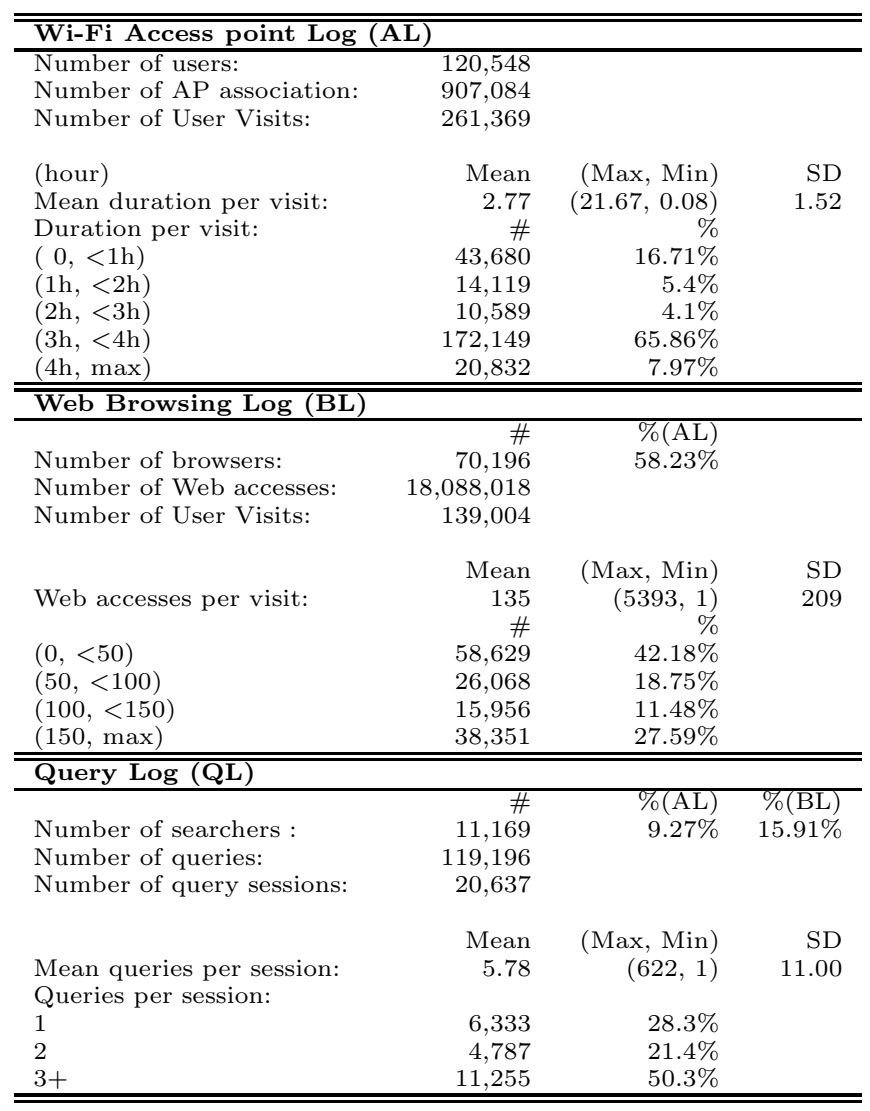

of QL), Bing (954, $0.8 \%$ of QL), Baidu (1086, 0.9\% of QL), AOL (43, $0.04 \%$ of QL) and ASK (50, $0.04 \%$ of QL).

The AL captures information about user physical behaviour through the following parameters: (1) users' location in the mall defined by the location of the Wi-Fi access point associated with the user's mobile device; (2) timestamp and duration of users' association with the access point. The BL includes the users' information behaviour, characterised by: (1) the timestamp of the Web request; (2) the Uniform Resource Locator (URL) of the requested Web page.

The QL was processed as follows: (1) search queries were treated as case insensitive; (2) a query term was defined as any unbroken string of characters in a query delimited by the whitespace symbol (U+0020); (3) Following [7, 4], sessions were defined as "a series of queries by a single user made within a small range of time". Following a recent study in Mobile Web search [12], we also use 30 minutes as the threshold for maximum session duration; (4) session length was defined as the number of queries in a session; (5) query length was defined as the number of terms in a query.

\section{PHYSICAL BEHAVIOUR AND WEB IN- FORMATION BEHAVIOUR}

In this study, we analyse the physical behaviour and Web information behaviour for indoor users by dividing them into four groups (number of unique users in brackets): (1) Open (112,322) denotes the users who appear during the mall's business hours (when shops are open); (2) Closed (8,226) denotes the users who appear when the mall is closed (there are some restaurants which are still open to the public after the retail part of the mall is closed); (3) Once $(80,900)$ denotes the users who appear only once in the collected period when the mall is open; (4) Many (31,422) denotes the users who appear more than once in the collected period when the mall is open. We observe that the majority of users come from the Open group, and there are more Once users than Many users. The Closed group was not split due to its small size. The majority of this group visited only once. Table 2 shows the comparison of the physical, browsing, and query activities among these user groups.

From AL, we can observe that (Table 1 and Table 2): (1) around $66 \%$ of users stay in the mall between three and four hours per visit, with $26 \%$ staying less than two hours and $8 \%$ exceeding three hours; (2) while Once users outnumber Many users, Many users tend to contribute more to the AL logs than Once users, due to their repeated visits to the mall (56.99\% of Many visited twice, $16.13 \%$ of them visited 3 times, and $26.88 \%$ visited 4 times or more); (3) Many stay in average longer than Once users (2.9 hour vs 2.4 hour), while Closed users stay longest (3.2 hour). Overall, mall users are most likely to stay in the mall for around 3 hours on average across different user groups.

From the BL, we observe that: (1) around $60 \%$ of Wi-Fi users in the AL actively browsed the Web, while the rest visited the mall but did not use the Web; (2) around $60 \%$ of these users accessed fewer than 100 URLs; (3) Many is the most Web-active user group (around 144 URLs per visit, significantly more than values for groups Once and Closed); (4) the number of BL logs from Closed is fairly low, with users only averaging 13 URLs per visit. Table 3 shows a breakdown of the content these users browse for on the Web. The top-10 popular Web categories of indoor browsing $^{1}$. While some of the top categories are related with retail activities (e.g. Business and Economy and Web Advertisements), many are related to social networking, infotainment and Personal information management (PIM). These results indicate that frequent visitors tend to be the most active on the Web, where they use diverse, and not only retail related content.

Consequently, we explore what indoor users search for on the Web. Is this content similar to their general search? From the QL, we find that: (1) around $10 \%$ of overall users in the $\mathrm{AL}(16 \%$ of browsers in BL) are searchers; (2) there is no big difference for indoor searching among different user groups in terms of the number of queries per session. (3) around $50 \%$ of the searchers issued three or more queries per session, which is different from general Web searchers [13]: most general Web searchers issue only one query per session. Potential explanations for these differences include: (a) people search differently in indoor retail spaces; (b) mobile search behaviours have changed since the publication of earlier studies; or (c) the behaviour of the modern interfaces has altered the patterns detectable in the logs (e.g., query suggestion by Google); (4) the top 10 indoor search categories (query-click) (Table 3, right column) are dominated by retail-related activities, e.g. Travel, Shopping,

\footnotetext{
${ }^{1}$ The Web page categories were generated through the public Webroot Content Classification Service bcws.brightcloud. com. Although DMOZ has higher accuracy, its coverage is to limited for our study. E.g. www.gumtree.com.au is not categorized in DMOZ but correctly categorized as shopping by BrightCloud.
} 
Table 2: Physical and Web activities by user group

\begin{tabular}{l|l|rrr}
\hline \hline \multirow{2}{*}{ Log } & \multirow{2}{*}{ statistics } & \multicolumn{2}{|c}{ Open } & \multirow{2}{*}{ Closed } \\
\cline { 3 - 4 } & & Once & Many & \\
\hline AL & No. of AP association & 257,444 & 530,028 & 119,612 \\
& \% of AP association & $28.4 \%$ & $58.4 \%$ & $13.2 \%$ \\
& mean duration (hour) & 2.4 & 2.9 & 3.2 \\
\hline BL & No. of Web accesses & $4,202,073$ & $12,738,140$ & $1,147,805$ \\
& \% of Web accesses & $23.2 \%$ & $70.4 \%$ & $6.3 \%$ \\
& mean Web access & 47.4 & 143.7 & 13.0 \\
\hline \multirow{2}{*}{ QL } & No. of Queries & 29,802 & 83,997 & 5,397 \\
& $\%$ of Queries & $25 \%$ & $70.5 \%$ & $4.5 \%$ \\
& mean queries per session & 5.50 & 5.87 & 5.85 \\
\hline \hline
\end{tabular}

Table 3: Top 10 Categories of Browsing and Query-Click

\begin{tabular}{l|l}
\hline \hline Browsing & Query-Click \\
\hline Social Networking (20\%) & Travel (12\%) \\
Content Delivery Networks (13\%) & Entertainment and Arts (9\%) \\
Computer and Internet Info (12\%) & Society (8\%) \\
Search Engines (11\%) & News and Media (8\%) \\
Business and Economy (10\%) & Shopping (8\%) \\
Personal Storage (5\%) & Reference and Research (7\%) \\
Web based email (3\%) & Social Networking (6\%) \\
Web Advertisements (3\%) & Business and Economy (6\%) \\
News and Media (3\%) & Personal Sites and Blogs (4\%) \\
Internet Portals (2\%) & Computer and Internet Info (4\%) \\
\hline \hline
\end{tabular}

Reference and Research and Business and Economy; (5) the search patterns of indoor users are different from their browsing activity, e.g. while Social Networking is the most popular browsing category (consistently with mobile Internet usage [3]); Travel is the most popular query-click category. Specifically, Travel makes $1.4 \%$ of browsing but $12 \%$ of searching and Social Networking takes $20 \%$ in browsing but only $6 \%$ in searching.

These differences imply that indoor browsing and searching should be treated differently to improve users' Web experience, because users are likely to satisfy different information needs via browsing and searching, respectively. For example, top browsing activities tend to be retail irrelevant, and may be accessed to satisfy common information needs not directly linked to the retail environment; while top search activities tend to be linked to retail, which may indicate tighter dependence on the spatial context of the retail environment. The role of specialised apps is also likely contributing to the difference in browsing vs. query traffic - users are more likely to use pre-installed specialised apps (e.g., Facebook) for social interaction rather than using the mobile version of the sites, and in any case the related Websites represent known target that need not be searched for. Moreover, while adult-related search was popular in general mobile search $[12,7,4]$, it is not popular in both indoor browsing and searching. We suspect one main reason is because the data were collected in a public indoor retail space.

Overall, we conclude that indoor users of the Many category tend to be much more active in the Web browsing. What indoor users browse for is different from what they search for. The Web categories of the searching activity are more related with the retail environment. Moreover, while the user groups differ in the number of URLs per visit in their Web browsing patterns (for a deeper analysis of BL, see [10].), the difference is small in the average number of search queries per session. We focus on the analysis of $\mathrm{QL}$ in the following Section 4.
Table 4: The session length $l$, mean $(l)$ and $|q|$ of indoor Web queries, general mobile Web queries and general Web queries. "-" means that the value was not listed in the corresponding paper.

\begin{tabular}{c|c|ccc|cc}
\hline \hline$l$ & \multirow{2}{*}{ Indoor } & \multicolumn{3}{|c|}{ General Mobile } & \multicolumn{2}{c}{ General Web Search } \\
& & Bing & Google & Euro & Dogpile06 & Dogpile05 \\
\hline 1 & $\mathbf{2 8 . 3 \%}$ & - & $68.0 \%$ & $45.0 \%$ & $52.8 \%$ & $53.9 \%$ \\
2 & $21.4 \%$ & - & $19.0 \%$ & $17.0 \%$ & $17.2 \%$ & $16.6 \%$ \\
$3+$ & $50.3 \%$ & - & $13.0 \%$ & $38.0 \%$ & $30.0 \%$ & $29.4 \%$ \\
\hline mean $(l)$ & 5.78 & 1.48 & 1.6 & 5.78 & - & 2.85 \\
\hline$|q|$ & 2.79 & 3.05 & 2.3 & 2.06 & 2.83 & 2.79 \\
\hline \hline
\end{tabular}

\section{INDOOR WEB SEARCH BEHAVIOUR}

In this section, we investigate Web search session length $l$ and query length $|q|$, which are two of the fundamental characteristics in Web search [1]. Table 4 shows the comparison of $l$, mean $(l)$ and $|q|$ among indoor queries, general mobile Web queries and general Web queries. We compare to past work on: Bing [12], Google Mobile [7], Euro Mobile [4], Dogpile '06 \& ' $05[5,9]$. These studies are selected because they study general mobile Web search or they define sessions in a similar way to our study. We observe that users tend to issue more queries than general mobile searchers and general Web searchers, but type queries in a similar way. The difference in query numbers again points to our hypothesis above, pointing to the recent introduction of query suggestion mechanisms in search engine interfaces. Overall, these statistics differ significantly for indoor queries from general mobile Web queries and general Web queries. Specifically, in the distribution of $l$ amongst indoor searchers, this user group has the lowest percentage of single query sessions, with the majority of indoor searchers submitting more than one query per session. We further focus on the regular patterns of the session length $l$ for indoor searchers in Section 5 .

\section{INDOOR WEB SEARCH PATTERNS}

We investigate the predictability of popular queries in indoor retail spaces, which has practical implications for the prediction of consumer behaviour.

\subsection{Search Patterns}

We first determine if the number of queries per session follows a two-parameter Inverse Gaussian (IG) distribution:

$$
p(l)=I G(l ; \mu, \lambda)=\left[\frac{\lambda}{2 \pi l^{3}}\right]^{\frac{1}{2}} \exp \frac{-\lambda(l-\mu)^{2}}{2 \mu^{2} l},
$$

where $\mu=E(l)=\frac{1}{m} \sum_{i=1}^{m} l^{i}$ denotes the mean ( $m$ is the total number of sessions in the $\log$ and $l^{i}$ is the length of $i$-th session), $\lambda=\frac{\mu^{3}}{\operatorname{var}(l)}$ is the shape parameter, and $\operatorname{var}(l)$ denotes the variance of $l$.

There are two theoretical underpinnings in IG distribution, which makes itself a good model of $l$ : 1) the indoor searching has a longer tail distribution (around half of the searchers submit more than two query per session). Thus, the consequent $\mu$ and $\lambda$ in IG can form a large and long tail; 2) the relatively larger head of the $l$ distribution can be fitted by the asymmetry feature of the IG distribution.

As Aijferuke et. al. [1] pointed out, when the sample size is large, the data can not be satisfactorily fitted using goodnessof-fit techniques, including chi-square $\chi^{2}$ test and Kolmogorov- 
Table 5: PCC $r$ Values (Google and Yahoo are investigated here, because they are the two most popular search engines on mobile devices in 2012 and 2013 netmarketshare.com and they are also the top two search engines in our QL)

\begin{tabular}{c|ccccc}
\hline \hline methods & All & Closed & Open & Google & Yahoo \\
\hline$C\left(q_{i}\right)$ & $\mathbf{0 . 9 2 8 0}$ & $\mathbf{0 . 7 5 6 0}$ & $\mathbf{0 . 9 2 6 9}$ & $\mathbf{0 . 9 2 1 1}$ & $\mathbf{0 . 9 3 6 4}$ \\
\hline \hline
\end{tabular}

Smirnov test. Following [6], we apply the coefficient of variation $R^{2}$ as an indication of the closeness of the fit.

To make a precise measurement of the quality of the theoretical fit, we perform a quantile-quantile analysis and observe that the $R^{2}$ value is $0.9597(P<0.001)$, which means that the theoretical fit describes almost all of the observed $l$ 's variance.

\subsection{Predicting Query Behaviour}

Here, we apply Eq. 1 to model and predict the popularity of queries in terms of query counts. We treat the collection of queries as a weighted directed graph $G=(Q, E)$, where the nodes $Q=\left\{q_{1}, \cdots, q_{n}\right\}$ denote the set of queries and weighted, directed $\operatorname{arcs} E=\left\{e_{i j}, \cdots, e_{h k}\right\}$ connect consecutive queries. If query $q_{j}$ is issued by a user immediately after query $q_{i}, e_{i j}$ is defined as $e_{i j}=\left(q_{i}, q_{j}\right)$. The weight $w_{i j}$ on $e_{i j}$ is defined as the fraction of users who search $q_{i}$ and then continue to $q_{j}: w_{i j}=\frac{o_{i j}}{\operatorname{deg}^{-}\left(q_{i}\right)}$, where $o_{i j}$ is the number of sequential co-occurrences from $q_{i}$ to $q_{j}$, and $\mathrm{deg}^{-}\left(q_{i}\right)$ is the in-degree of query $q_{i}$.

The prediction of the query popularity can then be modelled as the propagation process of query searching on the given graph $G$. Let $N_{l-1}^{j}$ denote the number of users who reach $q_{j}$ after searching $l-1$ queries, then the number of users who reach $q_{i}$ after searching $l$ queries is defined as: $N_{l}^{i}=\delta_{l} \sum_{j=1}^{n} w_{j i} N_{l-1}^{j}$, where $\delta_{l}=\frac{\int_{l}^{l+1} I G(\mu, \lambda)}{\int_{l-1}^{l} I G(\mu, \lambda)}$ and $I G(\mu, \lambda)$ is defined in Eq. 1. $\delta_{l}$ is interpreted as the fraction of users who search more than $l$ queries over users who search more than $l-1$ queries. This propagation process stops when the majority of modelled users stops searching.

The query log was chronologically split $80 \%-20 \%$ into continuous training and test sets, respectively. The training set is used to build the graph $G$, to estimate $\mu$ and $\lambda$ for the Inverse Gaussian distribution, and to initialize $N_{1}^{i}$ for all queries. The popularities of queries in the test are predicted by the method described above in terms of query counts, and the prediction of the query counts $C\left(q_{i}\right)$ for query $q_{i}$ is defined as: $C\left(q_{i}\right)=\sum_{k=1}^{l} N_{k}^{i}$. To measure the accuracy of prediction, we apply the Pearson Correlation Coefficient (PCC). As shown in Table 5, the predicted counts fit 4 out of 5 datasets with a $r$ value over 0.90 . The fit for the Closed subset is relatively low, a possible reason is that the search behaviour of the Closed group is less conditioned by the indoor environment (since shops are closed) and their Web search behaviour is therefore more varied and less predictable.

\section{CONCLUSION \& FUTURE WORK}

In this paper, we report the key characteristics of users' physical and Web behaviours in large indoor spaces. This shows an initial understanding of how mall visitors behave on the Web, and provides a chance to improve their Web and shopping experience. We have established that the indoor query behaviour is predictable in terms of query popularity, which has a practical application for the detection of search trends and recommendation services. In future work, we will further characterize and model location-based indoor search behaviour in order to better suggest contextualised results.

\section{Acknowledgement}

This research is supported by a Linkage Project grant of the Australian Research Council (LP120200413). We would like to thank Stefano Mizzaro for helpful discussions, and the anonymous reviewers and CIKM shepherd for valuable suggestions.

\section{REFERENCES}

[1] I. Ajiferuke, D. Wolfram, and F. Famoye. Sample size and informetric model goodness-of-fit outcomes: a search engine log case study. Journal of Information Science, 32(3):212-222, June 2006.

[2] S. Algethami. Dubai Mall welcomes more than 200,000 shoppers a day. Gulfnews, 2014.

[3] K. Church and N. Oliver. Understanding Mobile Web and Mobile Search Use in Today's Dynamic Mobile Landscape. In MobileHCI'11, pages 67-76, 2011.

[4] K. Church, B. Smyth, P. Cotter, and K. Bradley. Mobile information access: A study of emerging search behavior on the mobile Internet. ACM TWEB, 1(1), May 2007.

[5] B. J. Jansen, C. C. Ciamacca, and A. Spink. An Analysis of Travel Information Searching on the Web. Information Technology \& Tourism, 10(2):101-118, June 2008.

[6] S. Joo, D. Wolfram, and S. Song. Nonparametric estimation of search query patterns. In iConference 2013 Proceedings, pages 919-924, 2013.

[7] M. Kamvar and S. Baluja. A large scale study of wireless search behavior: Google mobile search. In CHI, pages 701-709, 2006.

[8] M. Kamvar, M. Kellar, R. Patel, and Y. Xu. Computers and iphones and mobile phones, oh my!: a logs-based comparison of search users on different devices. In $W W W$, pages 801-810, 2009.

[9] A. Kathuria, B. J. Jansen, C. Hafernik, and A. Spink. Classifying the user intent of web queries using k-means clustering. Internet Research, 20(5):563-581, 2010.

[10] Y. Ren, M. Tomko, K. Ong, Y. B. Bai, and M. Sanderson. The Influence of Indoor Spatial Context on User Information Behaviours. In $i$ - $A S C$ Workshop in conjunction with ECIR 2014, 2014.

[11] A. J. Ruiz Ruiz, H. Blunck, T. S. Prentow, A. Stisen, and M. B. Kjærgaard. Analysis Methods for Extracting Knowledge from Large-Scale WiFi Monitoring to Inform Building Facility Planning. In PerCom, pages 130-138. IEEE, 2014.

[12] Y. Song, H. Ma, H. Wang, and K. Wang. Exploring and Exploiting User Search Behavior on Mobile. In $W W W$, pages 1201-1212, 2013.

[13] A. Spink, D. Wolfram, M. B. Jansen, and T. Saracevic. Searching the Web: The Public and Their Queries. JASIST, 53(3):226-234, 2001. 